\title{
The competitive scenario of extra virgin olive oils in the modern distribution channel-an overview
}

\section{Opinion}

The present manuscript has come to analyze in general terms the main characteristic elements of modern distribution in relation to the olive oil italian market. This article is common in nature and presents itself as the basis for subsequent studies of in-depth analysis of the sector.

In Italian families the annual per capita consumption of olive oil ranges between 13-14 1/person (Italian Ministry of Agricolture, 2016). According the International Olive Oil Council, This is the highest value in the world, which underlines (Figure 1) the strategic value of the oil sector for the entire Italian agri-food system. One of the distinguishing features of the market for extra virgin olive oils is the presence of dualisms that characterize the offer of oils to the consumer. $^{1}$

The first dualism is represented by the simultaneous presence, in indirect distribution channels, for products of industrial packaging companies together with those of production and oil extraction companies, the first characterized by high concentration indexes and by the pursuit of economies of scale, the latter from an extreme variety of products and organoleptic characteristics. This is the result of the
Volume 6 Issue I - 2018

\author{
Francesco Diotallevi,Andrea Marchini \\ Department of Economics, Faculty of Food Sciences and \\ Agricultural, University of Perugia, Italy
}

Correspondence: Francesco Diotallevi, Department of Economics, Faculty of Food Sciences Agricultural, University of Perugia Borgo XX Giugno, 74 06।35-Perugia, Italy, +39 34789|2754, Email francesco.diotallevi@gmail.com Received: December 13, 2017 | Published: January 17,
2018

accentuated "maturity" of the sector which translates into a competitive complexity that is unrivaled in the other productive sectors. The result of this process is reflected in the simultaneous presence, in the market segment, of products that, although belonging to the same product category, have substantial consumer price differentials (from around 3 euro to over 20 euro liter) without an equally clear organoleptic differentiation of the product.

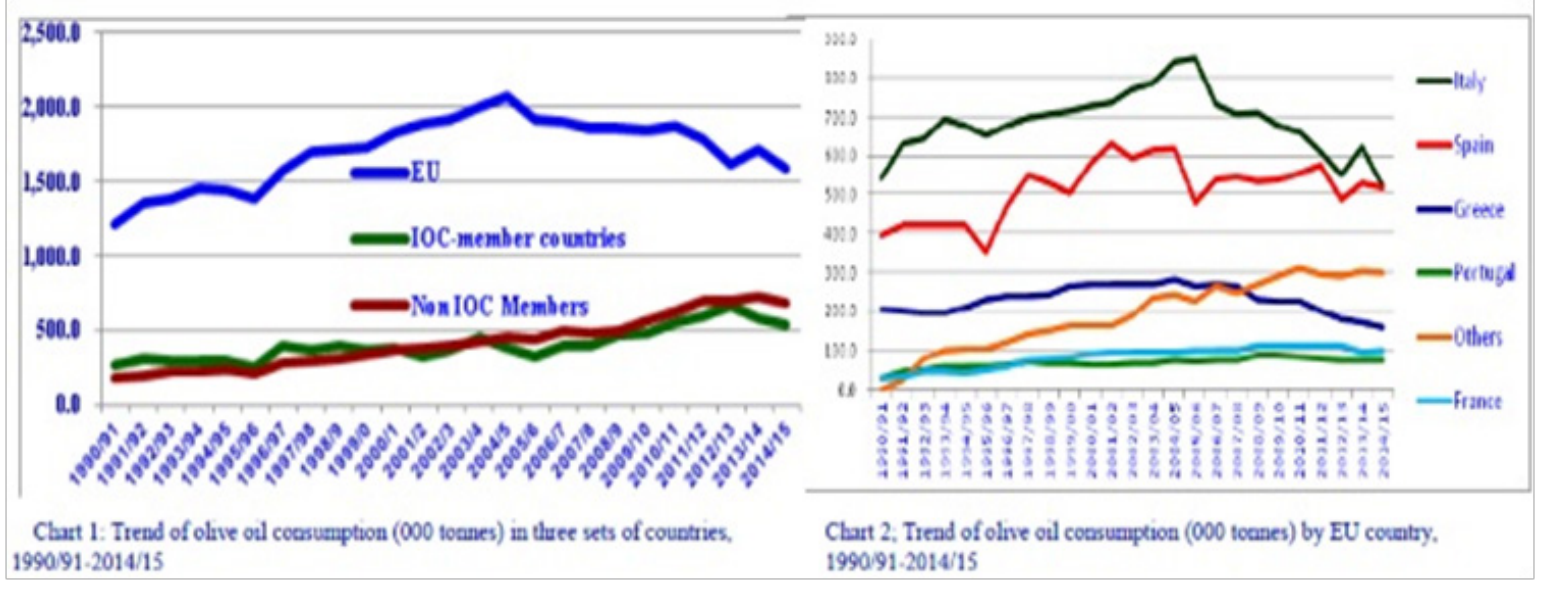

Figure I Trends of olive oil consumption.

All this feeds strong "informational asymmetries" in the exchange phase, especially for consumers who lack knowledge of the characteristics of the product, of the product, the production process and the production contexts. The second dualism is borne by the distribution forms with the simultaneous presence of direct and indirect sales channels. Contrary to what happens in many food production (milk, meat, etc.) where the evolution of the distribution channels has led to the progressive disappearance of the direct sales channel in favor of the most complex forms, for olive oils, and in particular For quality products, direct sales still represent an important channel for the opportunity that offers the producer to retain part of the added value of the product, avoiding the dispersion towards the downstream segments of the supply chain. The development of the information technology of the network allows today to enhance direct sales via the Web by increasing the capacity for "contact" of the producer and the possibility of integrating the distribution function even within small production companies with initiatives of the individual or their associated forms (farmers' markets, buying groups, etc.). The persistence and in many cases the development of direct sales forms is linked to the history and the productive and technological structure of the product: high number of small manufacturing companies in the area, extraction processes that can be carried out even in small or very small businesses, strong social integration and presence of a widespread culture of oil, etc.

The use of the indirect sales channel presents an intense articulation. On the one hand, the large-scale retail sector and on the other the Ho.Re.Ca. channel, represented by a complex of facilities 
(commercial and collective catering, specialized retail, catering, etc.) that are supplied both by individual producers and from wholesalers specialized in food service with exchanges assisted by multi-firm agents and/or concessionaires. The degree of market coverage is very different. The leading companies (Carapelli, Minerva, Farchioni, Monini) have national sales networks and have a widespread distribution throughout the territory, concentrating the exchanges within the Modern Distribution. The follower companies, even if they use the Modern Distribution, are generally focused in geographic areas, mostly circumscribed, operating through indirect networks locally sourced. Finally, small companies, together with direct sales in the "short market" have gradually expanded their distribution through the creation of new agencies, deposits and/or the use of dealers that allow their presence in many Italian regions, with a so-called "stain of leopard" distribution coverage. Despite the great variety of distribution forms, the Modern Distribution, which conveys over $60 \%$ of the consumption of extra virgin olive oil from the families, represents a significant field of study to understand the competitive dynamics. The following will provide an overview of the sales made in this channel, articulating the analysis by geographical area and commercial segment. In fact, given the vastness of the category of extra virgin oils, it is necessary to deepen the analysis within the same to investigate the performance of the new segments that characterize it. For this purpose the data were divided into three segments: the DOP/ IGP and Bio oils, the "100\% Italian" oils and finally the remaining extra virgin oils.

\section{Acknowledgements}

None.

\section{Conflict of interest}

None.

\section{References}

1. Diotallevi F, Stasi A, Marchini A. Price war: winner and losers in extravirgin olive oil. Proceedings of International Conference on Advanced Research in Scientific Areas (ARSA-2012); 2012. p. 388-398. 